\title{
Targeting on Morning Blood Pressure Surge
}

\section{Di Zhao ${ }^{\star 1}$ and Hui Liu ${ }^{2}$}

${ }^{1}$ Division of Hypertension, the First Affiliated Hospital, and College of Clinical Medicine of Henan University of Science and Technology, Luoyang ${ }^{2}$ Division of Endocrinology, Luoyang Central Hospital Affiliated to Zhengzhou University, Luoyang, China

\section{Abstract}

Morning blood pressure surge (MBPS) is an independent predictor of cardiovascular events. The most common definition of MBPS was a pre waking surge (mean blood pressure for 2 hours after wakeup minus mean blood pressure for 2 hours before wake-up). The sympathetic nervous system plays a major role in the regulation of BP variability. Asleep blood pressure has been shown to better reflect cardiovascular risk than awake BP in hypertensive patients. A possible link was found between disturbed sleep and MBPS. Exaggerated MBPS and increased low-grade inflammation independently increase the risk of stroke, while the relationship between exaggerated MBPS and the presence of silent cerebral infarct is slightly affected by low-grade inflammation. Furthermore, type 2 diabetes mellitus was an independent risk factor for increased MBPS. Serum uric acid, low density lipoprotein-cholesterol, anxiety disorder and so on may also involve in MBPS. Telmisartan was shown to normalize the circadian blood pressure pattern to a dipper profile in a larger proportion of patients than ramipril, and reduce early-morning systolic blood pressure surge in high-risk patients. Telmisartan alone or with HCTZ improved morning high blood pressure control and maintained a smooth high blood pressure profile throughout the day in a real-life setting. Furthermore, once-daily dosing of valsartan results in equally effective 24-hour blood pressure efficacy, regardless of dosing time. In total, future studies specifically designed to evaluate the real impact of MBPS on outcomes, as well as to define its optimal cutoff value, are required.

\section{Publication History:}

Received: October 16, 2017

Accepted: December 04, 2017

Published: December 06, 2017

\section{Keywords:}

Hypertension, Morning blood Pressure surge, Sympathetic nervous system, Diabetes, Telmisartan
Morning blood pressure surge (MBPS) is a special type of BP variability. MBPS is related to BP rise in the morning and BP fall at night during sleep. Its determinants are still poorly defined [1].

At present, seven different definitions of MBPS were identified; the most common was a pre waking surge (mean blood pressure for 2 hours after wake-up minus mean blood pressure for 2 hours before wake-up) [2].

Another important definition of MBPS was sleep-trough MBPS, which was calculated as morning blood pressure (average of 2 hours of readings after wake-up) minus the lowest night time reading (average of the lowest night time reading and the 2 adjacent readings before and after) [3]. A strong interrelationship between the MBPS and the nocturnal BP pattern exists [4].

Asleep BP has been shown to better reflect cardiovascular risk than awake BP in hypertensive patients. A possible link was found between disturbed sleep and MBPS. Clinicians should understand the importance of the modification of altered sleep pattern for reducing MBPS in non hypertensive participants [5]. In initially untreated subjects with hypertension, a blunted day-night BP dip was associated with a blunted MBPS and vice versa. In these subjects, a blunted MBPS was an independent predictor of cardiovascular events, whereas an excessive MBPS did not portend an increased risk of events [6]. However, the morning BP increase was attenuated during bed rest, suggesting that the adoption of an upright posture and/or physical activity in the morning contributes to the MBPS [7]. The clinically significant relationship between dipping and non-dipping patters, psychological traits and MSBP requires further investigation [8].

The riser pattern was a significant determinant of both blunted sleep-trough MBPS and blunted pre waking surge. The high prevalence of the riser pattern in patients with blunted MBPS may account for the cardiovascular risk previously reported in such patients [9].

It is generally accepted that the sympathetic nervous system plays a major role in the regulation of BP variability [10]. MBPS is associated with arterial stiffness and sympathetic baroreflex sensitivity, as well as vasoreactivity during orthostasis in hypertensive seniors
[10]. In untreated hypertension, MBPS has a direct relation with aortic stiffness, which is mediated by an increased average real variability of 24-hour systolic BP [11]. 24-hour BP rather than office BP is closely associated with cerebrovascular disease and/or poor physical and cognitive function [12]. The adverse effects of MBPS may be partly explained by its link with arterial stiffness, mediated by short-term systolic BP variability [11].

Exaggerated MBPS and increased low-grade inflammation independently increase the risk of stroke, while the relationship between exaggerated MBPS and the presence of silent cerebral infarct is slightly affected by low-grade inflammation [13], indicating lowgrade inflammation may affect MBPS. Furthermore, type 2 diabetes mellitus (T2DM) was an independent risk factor for increased MBPS, which was associated with central hemodynamic parameters [14]. Poor glycemic control [14,15] and insulin resistance [15] are independently associated with the occurrence of MBPS in T2DM patients, which might be significantly associated with endothelial dysfunction. Additionally sodium intake was associated with worse MBPS in T2DM [14].

Evaluation of hypertensive patients should also include an assessment of uric acid level and anthropometric measurements such as abdominal obesity. Hyperuricemia seems to be closely related to undesired BP patterns and this may signal to the clinician that an appropriate therapeutic approach is required [16]. Patients in the highest quartile stratified by elevated MBPS and serum uric acid level had a 3.55 odds of major cardiovascular events (CVEs) compared with patients in the lowest quartile. Moreover, serum uric acid is associated with MBPS and development of new CVEs [17].

"Corresponding Author: Dr. Di Zhao, Division of Hypertension, The First Affiliated Hospital, and College of Clinical Medicine of Henan University of Science and Technology, 24 Jinghua Street, Luoyang, 471003, China; E-mail: emaildizhao@163.com

Citation: Zhao D, Liu H (2017) Targeting on Morning Blood Pressure Surge. Int J Clin Res Trials 2: 119. doi: https://doi.org/10.15344/2456-8007/2017/119

Copyright: (c) 2017 Zhao, et al. This is an open-access article distributed under the terms of the Creative Commons Attribution License, which permits unrestricted use, distribution, and reproduction in any medium, provided the original author and source are credited. 
Several factors, such as low density lipoprotein-cholesterol, gammaglutamyl transferase activity, may also involve in MBPS [18,19]. The novel finding of a significant relationship between measures of MBPS and low density lipoprotein-cholesterol is an intriguing link between two major cardiovascular risk factors [18]. MBPS is independently associated serum gamma-glutamyltransferase activity in essential hypertensive patients [19]. In addition, anxiety disorder is associated with nocturnal and early morning hypertension, and may be a risk factor for cardiovascular events [20].

MBPS is an independent predictor of cardiovascular events [15]. In elderly treated hypertensive patients with normal achieved ambulatory BP, dippers with high MS and non dippers are at increased cardiovascular risk [21] and high MS of systolic BP predicts coronary events in dippers [22]. In older hypertensives, a higher MBPS is associated with stroke risk independently of ambulatory BP, nocturnal BP falls, and silent infarct [23]. Furthermore, in elderly treated hypertensive patients, high MS in systolic BP predicts stroke in dippers but not in non dippers. Non dippers are at high stroke risk with or without MBPS $>23 \mathrm{~mm} \mathrm{Hg}$ [24]. An exaggerated MBPS may be associated with stroke [2], higher hazard for death [25] and other cardiovascular events, but the threshold at which an MBPS becomes pathological is unclear [2].

However, this relationship was not confirmed by all the studies $[6,26]$. In a white population, MBPS was not found to be an independent predictor of cardiovascular death, all-cause death, or development of high cardiovascular risk even when appropriately assessed by measures that reflect its association with 24-hour BP variability [26]. These data suggest that MBPS is a complicated phenomenon with a somewhat unclear prognostic significance [4].

Clinic BP [27], morning and 24-hour ambulatory BP [27,28] control rates were low in treated hypertensive patients, including those with comorbidities, such as diabetes or left ventricular hypertrophy. In a retrospective analysis, telmisartan was shown to normalize the circadian BP pattern to a dipper profile in a larger proportion of patients than ramipril, and reduce early-morning systolic BP surge in high-risk patients, indicative of a cardioprotective effect [29]. Telmisartan alone or with HCTZ improved morning high BP control and maintained a smooth high BP profile throughout the day in a real-life setting $[28,30]$. Bedtime dosing of an angiotensin-converting enzyme (ACE)-inhibitor or angiotensin receptor blocker (ARB) shows a more sustained and consistent 24-hour antihypertensive profile, including greater night-time BP reduction [31-35].

Bedtime dosing of an ACE-inhibitor or ARB may improve the control of morning high BP, which may be helpful to reduce MBPS.

However, once-daily dosing of valsartan $320 \mathrm{mg}$ results in equally effective 24-hour BP efficacy, regardless of dosing time [31]. In addition, evening dosed nebivolol may confer some advantage over morning dosing in reducing pre waking systolic BP [34]. The mechanism may involve the vasodilation attributable to increased nitric oxide bioavailability [35].

In total, MBPS is a novel cardiovascular risk factor. Future studies specifically designed to evaluate the real impact of MBPS on outcomes, as well as to define its optimal cutoff value, are required (25).

\section{Competing Interests}

The authors declare that no competing interests exist.

\section{References}

1. Hoshide S, Kario K, de la Sierra A, Bilo G, Schillaci G, et al. (2015) Ethnic differences in the degree of morning blood pressure surge and in its determinants between Japanese and European hypertensive subjects: data from the ARTEMIS study. Hypertension 66: 750-756.

2. Sheppard JP, Hodgkinson J, Riley R, Martin U, Bayliss S, et al. (2015) Prognostic significance of the morning blood pressure surge in clinical practice: a systematic review. Am J Hypertens 28: 30-41.

3. Johansson JK, Niiranen TJ (2017) Morning surge and nocturnal dipping pattern: Two different entities or statistical gymnastics? J Clin Hypertens (Greenwich) 19: 1115-1116.

4. Suh M, Barksdale DJ, Logan JG (2014) Morning blood pressure surge and nighttime blood pressure in relation to nocturnal sleep pattern and arterial stiffness. J Cardiovasc Nurs 29: E10-E17.

5. Verdecchia P, Angeli F, Mazzotta G, Garofoli M, Ramundo E, et al. (2012) Day-night dip and early-morning surge in blood pressure in hypertension: prognostic implications. Hypertension 60: 34-42.

6. Morris CJ, Hastings JA, Boyd K, Krainski F, Perhonen MA, et al. (2013) Day/night variability in blood pressure: influence of posture and physical activity. Am J Hypertens 26: 822-828.

7. FitzGerald L, Ottaviani C, Goldstein IB, Shapiro D (2012) Effects of dipping and psychological traits on morning surge in blood pressure in healthy people. J Hum Hypertens 26: 228-235.

8. Fujiwara T, Tomitani N, Sato K, Okura A, Suzuki N, et al. (2017) The relationship between a blunted morning surge and a reversed nocturnal blood pressure dipping or riser pattern. J Clin Hypertens (Greenwich) 19: 1108-1114.

9. Cho JS, Ihm SH, Jang SW, Chung WB, Choi YS, et al. (2014) Negative association between plasma aldosterone concentration/plasma renin activity and morning blood pressure surge in never-treated hypertensive patients. Clin Exp Hypertens 36: 195-199.

10. Okada Y, Galbreath MM, Shibata S, Jarvis SS, Bivens TB, et al. (2013) Morning blood pressure surge is associated with arterial stiffness and sympathetic baroreflex sensitivity in hypertensive seniors. Am J Physiol Heart Circ Physiol 305: 793-802

11. Pucci G, Battista F, Anastasio F, Schillaci G (2017) Morning pressor surge, blood pressure variability, and arterial stiffness in essential hypertension. $J$ Hypertens 35: 272-278.

12. Yano $Y$, Kario K (2012) Nocturnal blood pressure, morning blood pressure surge, and cerebrovascular events. Curr Hypertens Rep 14: 219-227.

13. Shimizu M, Ishikawa J, Yano Y, Hoshide S, Shimada K, et al. (2011) The relationship between the morning blood pressure surge and lowgrade inflammation on silent cerebral infarct and clinical stroke events. Atherosclerosis 219: 316-321

14. Yoda K, Inaba M, Hamamoto K, Yoda M, Tsuda A, et al. (2014) Association between glycemic control and morning blood pressure surge with vascular endothelial dysfunction in type 2 diabetic patients. Diabetes Care 37: 644650.

15. Afsar B, Elsurer R (2014) The relationship between central hemodynamics, morning blood pressure surge, glycemic control and sodium intake in patients with type 2 diabetes and essential hypertension. Diabetes ResClin Pract 104: 420-426.

16. Tutal E, Sayın B, Ertugrul DT, Ibis A, Sezer S, et al. (2013) Is there a link between hyperuricemia, morning blood pressure surge, and non-dipping blood pressure pattern in metabolic syndrome patients? Int $\mathrm{J}$ Nephrol Renovasc Dis 6: 71-77.

17. Turak O, Afsar B, Ozcan F, Canpolat U, Grbovic E, et al. (2014) Relationship between elevated morning blood pressure surge, uric acid and cardiovascular outcomes in hypertensive patients. J Clin Hypertens (Greenwich) 16: 530-535.

18. Martin CA, Cameron JD, Head GA, Chen SS, Eikelis N, et al. (2013) The morning blood pressure surge is related to serum cholesterol. J Hum Hypertens 27: 315-320.

19. Elsurer R, Afsar B (2015) Morning blood pressure surge is associated with serum gamma-glutamyltransferase activity in essential hypertensive patients. J Hum Hypertens 29: 331-336.

20. Kayano H, Koba S, Matsui T, Fukuoka H, Toshida T, et al. (2012) Anxiety disorder is associated with nocturnal and early morning hypertension with or without morning surge: ambulatory blood pressure monitoring. Circ $J$ 76: 1670-1677. 
21. Pierdomenico SD, Pierdomenico AM, Coccina F, Lapenna D, Porreca E, et al. (2017) Prognostic Value of Nondipping and Morning Surge in Elderly Treated Hypertensive Patients with Controlled Ambulatory Blood Pressure. Am J Hypertens 30: 159-165.

22. Pierdomenico SD, Pierdomenico AM, Di Tommaso R, Coccina F, Di Carlo S, et al. (2016) Morning Blood Pressure Surge, Dipping, and Risk of Coronary Events in Elderly Treated Hypertensive Patients. Am J Hypertens 29: 39-45.

23. Kario K, Pickering TG, Umeda Y, Hoshide S, Hoshide Y, et al. (2003) Morning surge in blood pressure as predictor of silent and clinical cerebrovascular disease in elderly hypertensives: a prospective study. Circulation 107: 1401-1406.

24. Pierdomenico SD, Pierdomenico AM, Cuccurullo F (2014) Morning blood pressure surge, dipping, and risk of ischemic stroke in elderly patients treated for hypertension. Am J Hypertens 27: 564-570.

25. Amodeo C1, Guimarães GG, Picotti JC, dos Santos CC, Bezzerra Fonseca $\mathrm{KD}$, et al. (2014) Morning blood pressure surge is associated with death in hypertensive patients. Blood Press Monit 19: 199-202.

26. Bombelli M, Fodri D, Toso E, Macchiarulo M, Cairo M, et al. (2014) Relationship among morning blood pressure surge, 24-hour blood pressure variability, and cardiovascular outcomes in a white population. Hypertension 64: 943-950.

27. Parati G, Bilo G, Redon J, SURGE Steering Committee (2013) Morning and smooth 24-h ambulatory blood pressure control is not achieved in general practice: results from the SURGE observational study. J Hypertens 31: 616-623.

28. Parati G, Bilo G, Redon J, SURGE Steering Committee (2013) The effects of telmisartan alone or with hydrochlorothiazide on morning and 24-h ambulatory BP control: results from a practice-based study (SURGE 2). Hypertens Res 36: 322-327.

29. Gosse P, Schumacher H (2014) Effect of telmisartan vs. ramipril on 'dipping' status and blood pressure variability: pooled analysis of the PRISMA studies. Hypertens Res 37: 151-157.

30. Redon J, Bilo G, Parati G, Surge Steering Committee (2013) The effects of telmisartan alone or in combination with hydrochlorothiazide on morning home blood pressure control: the SURGE 2 practice-based study. Blood Press 22: 377-385

31. Zappe DH, Crikelair N, Kandra A, Palatini P (2015) Time of administration important? Morning versus evening dosing of valsartan. J Hypertens 33 385-392.

32. Hermida RC, Ayala DE (2009) Chronotherapy with the angiotensinconverting enzyme inhibitor ramipril in essential hypertension: improved blood pressure control with bedtime dosing. Hypertension 54: 40-46.

33. Hermida RC, Ayala DE, Ferna'ndez JR, Calvo C (2007) Comparison of the efficacy of morning versus evening administration of telmisartan in essential hypertension. Hypertension 50: 715-722.

34. Acelajado MC, Pisoni R, Dudenbostel T, Oparil S, Calhoun DA, et al. (2012) Both morning and evening dosing of nebivolol reduces trough mean blood pressure surge in hypertensive patients. J Am Soc Hypertens 6: 66-72.

35. Okamoto LE, Gamboa A, Shibao CA, Arnold AC, Choi L, et al. (2014) Nebivolol, But Not Metoprolol, Lowers Blood Pressure in Nitric OxideSensitive Human Hypertension. Hypertension 64: 1241-1247. 